\title{
Registration of retinal images by a MAS-ICP approach - a preliminary study
}

\author{
Carla Pereira ${ }^{1}$, Nelson Martins ${ }^{1}$, Luís Gonçalves ${ }^{2}$, Manuel Ferreira ${ }^{1}$ \\ ${ }^{1}$ Industrial Electronics Department, University of Minho, Campus de Azurém, 4800-058 Guimarães \\ ${ }^{2}$ Ophthalmology Service, Hospital Center of Alto Ave, EPE - Unit of Guimarães, Portugal \\ mjf@dei.uminho.pt (PI)
}

\begin{abstract}
Diabetic retinopathy has been revealed as the most common cause of blindness among people of working age. For monitoring the pathology image registration algorithms applied to retinal images is very useful. In this work, a novel vessel-based retinal image registration approach is proposed. The segmentation of the vasculature is performed by a multi-agent system model. All these information is then used in a Robust Point Matching Iterative Closest Point algorithm improved by a Region Bootstrap approach. With this preliminary study, the novelty of integrating all these algorithms for image registration preceded by a multi-agents system for image edges detection seems to be efficient for temporal retinal image registration. Consequently, a system developed on basis of this approach could help in screening programs for the diabetic retinopathy prevention.
\end{abstract}

Index Terms - Diabetic retinopathy; Fundus images; Image registration; Iterative Closest Point; Multi-agents system.

\section{CONTEXT}

Diabetic retinopathy (DR) is a specific microvascular complication of diabetes mellitus and has been revealed as the most common cause of blindness among people of working age. One contribution of image processing to the diagnosis of $\mathrm{DR}$ is for monitoring the pathology. For assessing the evolution of the DR, specialists have to compare images taken at different medical examinations. In order to facilitate this very-time consuming task due to distortions between images, several registration algorithms have been proposed in literature [1]-[4]. Deng et al. [4] divide the approaches in two categories: vessel-based and nonvessel-based, since the blood vessels features are frequently used as a basis for image matching. Most of the vessel-based algorithms do not use all the information for the registration step. Instead, they reduce the information using only bifurcations and cross points. These kinds of methods have less computation time but have the problem of being more sensitive to the quality of segmentation. Moreover, they overlook some information that might be important. In this preliminary study, a vessel-based retinal image registration is proposed. This approach is compounded of two main steps: edge detection of the retinal blood vessels by a multi-agent system (MAS) model, and image matching by the Robust Point Matching Iterative Closest Point (RPM_ICP) algorithm.
The main difficulties in accurately segment the vessels are due to the presence of pathologies, noise, the low contrast between vasculature and background, the variability of vessels width, brightness and shape. To solve this problem of variability, it is important to locally adapt interpretations on the image instead of applying only one algorithm on the entire image. A multi-agent system (MAS) is thus proposed as a solution since agents allow the cohabitation of several algorithms [5] As far as known, applying MAS to retinal images is a novelty. In this work, the idea is to use some image processing algorithms as concrete perception and action tools for defining autonomous agents that will locally interact among themselves and with the environment (the image) at the micro level. Then a global behavior will emerge from these micro level interactions, which in this case will be the segmentation of the blood vessels.

Using all information from segmentation lead to some challenges such as defining the correct matching of large amounts of points and handling the points without correspondence (outliers). To overtake this problem a new algorithm based on ICP is proposed, the Region Bootstrap RPM_ICP. An ICP based algorithm is used because it is a point-based method, which is almost immune to the nonuniform illumination, and due its versatility. To improve the results of the ICP, instead of a simple minimum distance criterion, a RPM algorithm was used to handle the points leading to a better correspondence.

\section{GOALS}

This work has been implemented during a large project which central goal is the development of a computer aided diagnosis system to be applied in regular screening programs to detect DR. The task of segmenting the blood vessels is very useful for two situations in this project: aiding in the DR diagnosis; providing the localization of landmark points needed for image registration. In this preliminary study, the segmentation of the blood vessels by a MAS approach was used for superimposing retinal images by the ICP algorithm. This step is very helpful for the temporal evaluation of the DR.

\section{TEAM AND INSTITUTIONS}

The project has been developed in the Industrial Electronics Department from University of Minho. For the medical

\footnotetext{
$2^{\text {nd }}$ Portuguese Meeting in Bioengineering, February 2012

Portuguese chapter of IEEE EMBS

Rectory of the University of Coimbra
} 
purposes the team has the contribution of the Hospital Center of Alto Ave, EPE - Unit of Guimarães. Moreover, for understanding the MAS, researchers of the CreSTIC from University of Champagne-Ardenne gave a great contribution.

\section{IMPLEMENTATION}

The proposed approach uses a MAS model to detect the retinal blood vessels edges resulting from a preprocessing phase. This preprocessing phase consists of a group of conventional image processing algorithms and provides the information (environment) for the MAS model reconstructs and re-organizes them. Then the information from the previous segmentation is used for a region bootstrap non-rigid ICP algorithm, in order to proceed with the image registration.

\section{A. Preprocessing}

For this step of the approach, the methods used in the preprocessing phase of work developed by Niemeijer et al. [6] were first employed. The resultant image had no intensity variation in the background across the image and the bright structures were eliminated. A small Gaussian filter was then applied to attenuate the high frequency noise.

In order to remove the noise of the fundus image while preserving the edges it was then applied the Kuwahara filter [7]. Finally to the image resulted from the last step a modified version of the Kirsch filter was employed [5]. The Kirsch filtering is a method for enhancing the edges in an image and this improved version enables to detect edges with a two pixels thickness of which the external edge is represented by a positive or negative value, whereas the internal edge has an opposite value (Fig. 1 a)). This facilitates the detection process of the MAS model since the gradient of the blood vessels reveals a specific pattern (Fig. 1 b)) as they can be represented by series of two parallel linear segments. Thus, the agents search the edges of the blood vessels by looking for this specific pattern of the gradient.

\section{B. Multi-agent approach}

The MAS is composed by a set of agents and their environment. The environment contains the green plane image in which each pixel contains the grey level of intensity and a boolean value defining if the pixel has already been explored by an agent. When located in the environment the agents perceive the Kirsch gradient which defines a right visible edge. The agents are of several kinds with different behaviors according to their current state and perception. The search agents (SA) explore the environment looking for the edges of the blood vessels; the following agents (FA) follow a detected edge; the node agents (NA) construct segments; the end agents make a confirmation if the segment belongs to a vessel edge.

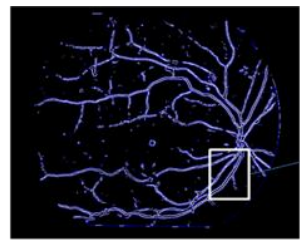

a)

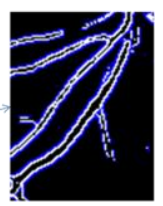

Figure 1 - a) Resultant image of the modified Kirsch filter where the blue and white pixels represent negative and positive gradient values, respectively. b) Expanded version of a section of image a), where is possible to see a characteristic pattern in the vessel gradient values.

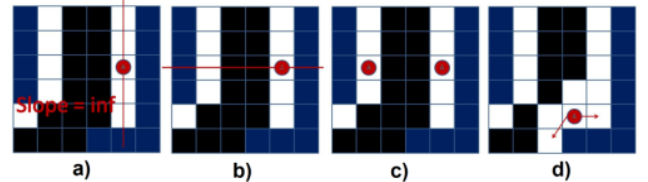

Figure 2 - The SA (red circle) behavior. a) It calculates the slope of the line to determine the perpendicular line (b). Then it verifies the gradient values profile and as the pixel belongs to a vessel pattern it launches another SA (c); d) Possible directions (red arrows) that the agent has to follow according to the search agents' restrictions

As the SA are looking for blood vessels, it was made a list of the white points $\left(\mathrm{L}_{\mathrm{p}}\right)$ of the Fig. 1. a), that corresponds to the positive values of the gradient. The SA is launched on one point randomly chosen from $\mathrm{L}_{\mathrm{p}}$ and verifies if it is located on a pixel: (1) not visited yet by another agent; (2) corresponding to the vessel pattern. If it verifies the two conditions it determines the possible directions to follow, launches a NA on its position and moves to another white point, also randomly chosen. Moreover, it launches another SA on the parallel line to the one where it is initially located (Fig 2 a)-c)). For the determination of the directions to follow the agents look for the white points in their 8-neighbooring having a blue point in the 4neighbooring. This blue point also has to belong to the 8neighboors of the target pixel (Fig. 2 d)). The initially launched SA stops its behavior and disappears when all the points of $\mathrm{L}_{\mathrm{p}}$ were analyzed.

The NA launches FA in the directions given by the launcher agent (SA or FA) and establishes segments with that.

The FA is linked with a NA which does not move. To construct the edge, it moves pixel per pixel and it stores the pixel position on it is located. The objective is to make sure that each position characterizing the edge portion which separates it from its neighbor can be approximated (using a threshold) by the segment connecting the two agents. The FA move by determining the possible directions to follow as the SA do. If the FA has just one direction to follow, it checks if the segment formed between the position of its neighbor and its positions is a valid segment, and if it is not, it launches another $\mathrm{NA}$ on its position and disappears. If two FA find each other when they are moving in a line vessel but in opposite directions, they ask for a simple fusion process.

When there is no direction to follow an EA is launched. The behavior of the EA is to check if it is located on a blood vessel edge by analyzing the green intensity profile on the perpendicular direction to its segment. It just verifies if the profile is similar to a Gaussian shape as the blood vessels should be. If the profile does not fit a Gaussian curve, the EA disappear with its segment. If the profile fits a Gaussian curve the EA stops its behavior.

\section{Region Bootstrap non-rigid ICP}

The registration phase starts with a pre-processing stage leading to a more robust initialization. The next stage is the transformation calculation. In this step three incorporated cycles are used. The first one is responsible for adding points to the RPM_ICP algorithm and is called Region Bootstrap. The second one is responsible for calculate the quadratic transforms matrixes for each region and is called RPM_ICP. 
The inner one is a cycle used to optimize the correspondence between points while re-estimate the transformation parameters. The final step consists in applying the calculated transformations to the target image for obtaining the registered image.

1) Pre-registration and point filtering: The first step for the image registration process consists in computing a translation based on the optic disk center of both images. The aim of this step is to make a coarse approximation of the transformation, reducing significantly the computation time. Moreover, it prepares the points to be easily used in the Region Bootstrap process and helping ICP preventing it to get stuck in local minimums. The position of the optic disk is obtained manually.

After this pre-registration phase, the points outside the resulting overlapping area are removed. This filtering reduces significantly the number of outliers without lost of important information, which will lead to a faster and robust algorithm.

2) Bootstrap Region: Since the number of points in the both clouds is too high, it is time consuming to process all of them at the same time. In [1] a method called "Bootstrap Region" tries to smooth this problem. The idea is to start with a small amount of points and calculate the transform parameters; add then more points and calculate a new transformation, until all points are used. In the present work, we start with the points inside a circular area centered at the optic disk. At each cycle new points are added, increasing the circle radius, and a transformation is calculated. At the end we have $\mathrm{N}$ transforms which are applied to the target image in order to get the registered image.

The calculation of the transform parameters is done in two steps: the robust point matching and the parameters reestimation (RPM_ICP). These two steps are repeated iteratively until convergence is achieved.

a) Robust Point Matching: In order to match the points from both clouds a correspondence matrix is used. This matrix compares all points from one cloud with all points from the other. The main idea is to give a score to every single correspondence and then use this information to get an approximated correspondent point. To handle the points without correspondence (outliers) an extra column and row are added as shown in Fig.3.

Note that achieving a binary matrix early in the matching process is unlikely since the correspondence is not known. So the problem must be reviewed in order to the matrix can handle correspondences properly. In [2] the notion of softassign and annealing is introduced. Therefore, instead of given a binary value at each pair, we give a score based on the potential of the correspondence based on equation (1).

\begin{tabular}{|c|c|c|c|c|c|c|}
\hline$M(i, j)$ & $x_{1}$ & $x_{2}$ & $x_{3}$ & $x_{4}$ & $x_{5}$ & Outliers \\
\hline$v_{1}$ & 0 & 0 & 0 & 0 & 0 & 1 \\
\hline$v_{2}$ & 0 & 1 & 0 & 0 & 0 & 0 \\
\hline$v_{3}$ & 0 & 0 & 0 & 0 & 1 & 0 \\
\hline$v_{4}$ & 1 & 0 & 0 & 0 & 0 & 0 \\
\hline Outliers & 0 & 0 & 1 & 1 & 0 & \\
\hline
\end{tabular}

Figure 3 - Example of a final correspondence matrix $M(i, j)$ where points $\left[\begin{array}{lll}x_{3} & x_{4} & v_{1}\end{array}\right]$ are outliers and $\left[\begin{array}{ll}x_{1} & v_{4}\end{array}\right],\left[\begin{array}{ll}x_{2} & v_{2}\end{array}\right]$ are matched points.

$$
M(i, j)=\frac{1}{T} * e^{-\frac{\left(x_{i}-f\left(v_{j}\right)\right)^{r} *\left(x_{i}-f\left(v_{j}\right)\right)}{2 T}}
$$

Where $x_{i}$ is the reference point, $f\left(v_{j}\right)$ is the result of applying the transform to the moving points. The $\mathrm{T}$ term represents the entropy temperature and can be seen as a control variable. It can be notice that for high values of $\mathrm{T}$ the score is less sensible to the distance between the points leading to a less accurate correspondence. As the iterations goes on, the temperature is decreased and the correspondence gets more and more accurate. At the end it is expectable to have a binary matrix. The temperature is lowered by a factor between $1 \%$ and $10 \%$ each cycle until it reaches a definitive threshold and the iterative process is finished.

b) Transform Parameter Re-estimation: Attending to the shape of the eye, it is clear that a non-rigid registration must be applied. There are several non-rigid methods from which we choose the quadratic transformation used by Stewart et al [1] (equation 2). This kind of transformation has less degree of freedom (DoF) than a diffeomorphic method. However, since the eye deformation represents more a global distortion instead of a localized one, the $12 \mathrm{DoF}$ is more suitable for the problem.

$$
\left[\begin{array}{l}
f_{(x)} \\
f_{(y)}
\end{array}\right]=\left[\begin{array}{llllll}
a_{11} & a_{12} & a_{13} & a_{14} & a_{15} & a_{16} \\
a_{21} & a_{22} & a_{23} & a_{24} & a_{25} & a_{26}
\end{array}\right] *\left[\begin{array}{c}
p_{x} \\
p_{y} \\
p_{x}{ }^{2} \\
p_{y}{ }^{2} \\
p_{x} p_{y} \\
1
\end{array}\right]
$$

In equation (2) $f(x)$ and $f(y)$ represent the result of the application of the transformation $\mathrm{f}$ (coefficients $a_{i j}$ ) to the points $p_{x}$ and $p_{y}$. For getting the $a_{i j}$ parameters, the correspondence obtained from the RPM step is used. Note that, at this point, $M$ is a continuous defined matrix with $M €$ [0 1]. In that way, the correspondence could not be done "one to one", and consequently we propose a correspondence "one to all" as shown equation (3).

$$
v_{i}{ }^{\prime}=\sum_{j=0}^{\max (j)} M(i, j) * v_{j}
$$

Where $v_{i}$ ' is the approximated assigned point of $x_{i}$. With all the correspondences assigned, a Single Value Decomposition (SVD) algorithm is used to calculate the new parameters that are used in the RPM to actualize the scores.

Note that RPM and parameters re-estimation steps are repeated 5 times before lowering $\mathrm{T}$. When threshold is reached, the last transform matrix $f$ is used to register the image.

\section{RESULTS}

The performance of the edge reconstruction by the proposed MAS model depends directly on the image processing algorithms used in the preprocessing phase. It also depends of how the system interprets information resulting from this first phase of the approach. To observe the performance of the MAS approach, it is important to compare the resulting image with the information detected by the Kirsch filter. 
Results of the proposed approach applied to retinal images are shown in Fig. 4. These illustrate the green planes of the images and the segmentation performed by the MAS. By analyzing Fig. 1 a) and Fig. 4 (left one, below) it can be observed that the MAS reconstructed the edges of the most part of the vessels, especially of the thickest ones. Furthermore, they eliminated a large part of the pixels detected by the Kirsch filter that do not belong to the vessels. However, the MAS also cleaned some pixels belonging to the thinnest vessels, and some of these vessels were not detected at all, affecting the sensitivity values (Fig. 4). However, for registration purposes this is not meaningful since a good detection of the largest vessel is sufficient.

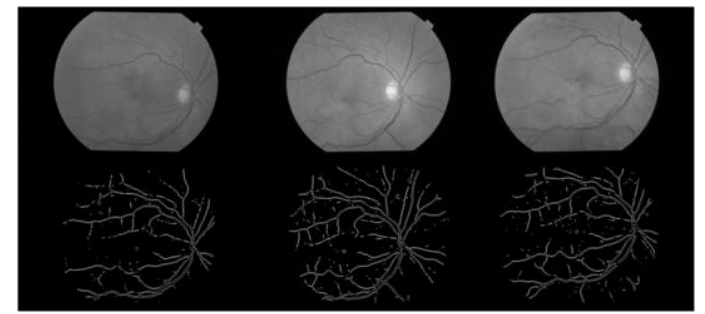

Figure 4 - Original green plane image (top) and the corresponding resulting images from the MAS approach (below).

Attending to the registration results, it is possible to see that the algorithm was able to register the image. In figure 5 it is shown the reference image (top) and two processed images (below). A grid was added to simplify the image comparison. It is possible to see that the non-overlapping areas were registered despite of the lack of segmented vessels, due the global quadratic transform. This is a good result, but must be treated carefully because there is no guarantee that the registration in this area is done properly.

\section{CONCLUSIONS}

In this study, a novel image registration approach for being applied in retinal images is proposed. As it is a vessel-based method, the first phase consists in segmenting the blood vessels by a MAS model, where agents enrich a traditional edge detector algorithm allowing local processing adaptation and cooperative behaviors. The system is able to represent the vessel edges present on fundus image through segments. Then, a Robust Point Matching Iterative Closest Point algorithm improved by a Region Bootstrap approach is used. Using this combination of techniques with the ICP allows the use of all the points in a robust and efficient way, instead of using just a few points and taking the risk of losing some important information. With the results of this preliminary study, it seems that the integration of all these algorithms for image registration preceded by a multi-agents system for image edges detection could be an efficient approach for temporal retinal image registration. Consequently, a system developed on basis of this approach could help in screening programs for the diabetic retinopathy prevention.

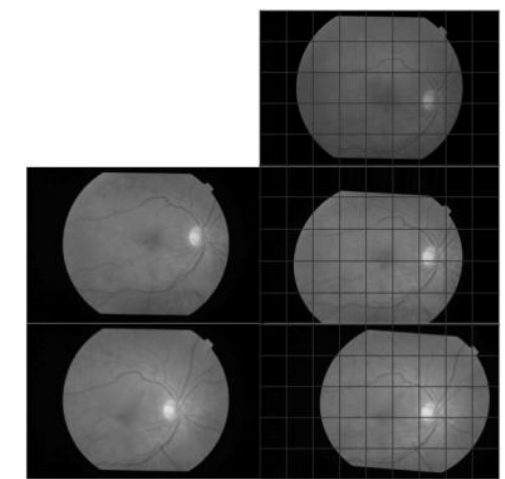

Figure 5 - Reference image (top) and two registered images (middle and bottom right) and their respective unregistered images (middle and bottom left)

\section{Planned DeVelopments}

As future work, using an improved version of the society of agents with some knowledge a priori about the retina proprieties can benefit the segmentation of the vasculature. But as for registration purpose this is not the most important, the planned development are more related to the registration step. For that, several improvements must be done, starting with parameters reduction and optimization. The use of parallel computing can be explored to reduce the necessary time for registering each image, which is still very long. Moreover, more validations in a large database must be done in order to test the robustness of the method.

\section{ACKNOWLEDGMENT}

C. P. thanks the Fundação para a Ciência e Tecnologia (FCT), Portugal for the Ph.D. Grant SFRH/BD/ 61829/2009.

\section{REFERENCES}

[1] C. Stewart, C. Tsai, B. Roysam, "The dual-bootstrap iterative closest point algorithm with application to retinal image registration," IEEE Trans. Med. Imag., Vol. 22, no. 11, pp. 1379-1394, 2003

[2] H. Chui, A. Rangarajan, “A new point matching algorithm for non-rigid registration, " Computer Vision and Image Understanding, Vol. 89, no. 2-3, pp. 114-141, 2003.

[3] B. Fang, Y. Tang, "Elastic registration for retinal images based on reconstructed vascular trees," IEEE Trans. On Biomedical Engineering, Vol. 53, no. 6, pp. 1183-1187, 2006

[4] K. Deng, J. Tian, J. Zheng, X. Zhang, X. Dai, M. Xu, "Retinal fundus image registration via vascular structure graph matching, "International Journal of Biomedical Imaging, 2010.

[5] J. Mahdjoub, Z. Guessoum, F. Michel, M. Herbin, “ A multi-agent approach for the edge detection in image processings," Proceedings of the 4th European Workshop on Multi-Agent Systems, Lisbon, Portugal, 2006.

[6] M. Niemeijer, B. van Ginneken, J. Staal, M. Suttorp-Schulten, M.D. Abràmoff, "Automatic detection of red lesions in digital color fundus photographs,” IEEE Trans. Med. Imag., Vol. 24, no. 5, pp. 584-592, 2005.

[7] M.Kuwahara, K. Hachimura, S. Eiho, M. Kinoshita, "Digital Processing of Biomedical Images,” Plenum Press, pp. 187- 203, New York, NY, 1976 